Correction

\title{
Correction: MALDI-IMS combined with shotgun proteomics identify and localize new factors in male infertility
}

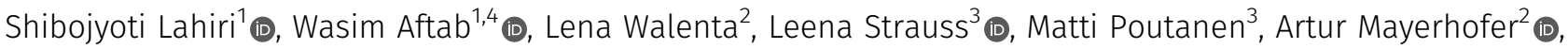
Axel Imhof ${ }^{1}$ (1)

${ }^{1}$ Biomedical Center, Protein Analysis Unit, Faculty of Medicine, Ludwig-Maximilians-Universität München, Planegg-Martinsried, Germany ${ }^{2}$ Biomedical Center, Cell Biology-Anatomy III, Ludwig-Maximilians-Universität München, Planegg-Martinsried, Germany Institute of Biomedicine, Research Centre for Integrative Physiology and Pharmacology and Turku Center for Disease Modeling, University of Turku, Turku, Finland ${ }^{4}$ Graduate School for Quantitative Biosciences (QBM), Ludwig-MaximiliansUniversität Munich, Munich, Germany

DOI https:// doi.org/10.26508/lsa.202101015 | Received 8 January

2021 | Accepted 8 January 2021 | Published online 21 January 2021

See original article: MALDI-IMS combined with shotgun proteomics identify and localize new factors in male infertility, 4(3), 2021.

LSA regrets that in the original version of this article, the labels for mass were mistakenly listed as kilodaltons (KD) instead of dalton (Da). The errors appear only in PDFs downloaded before January 7, 2021.

License: This article is available under a Creative Commons License (Attribution 4.0 International, as described at https://

creativecommons.org/licenses/by/4.0/). 